\title{
No quiet surrender: molecular guardians in multiple sclerosis brain
}

\author{
Lawrence Steinman \\ Department of Neurology and Neurological Sciences, Stanford University, Stanford, California, USA.
}

\begin{abstract}
The brain under immunological attack does not surrender quietly. Investigation of brain lesions in multiple sclerosis (MS) reveals a coordinated molecular response involving various proteins and small molecules ranging from heat shock proteins to small lipids, neurotransmitters, and even gases, which provide protection and foster repair. Reduction of inflammation serves as a necessary prerequisite for effective recovery and regeneration. Remarkably, many lesion-resident molecules activate pathways leading to both suppression of inflammation and promotion of repair mechanisms. These guardian molecules and their corresponding physiologic pathways could potentially be exploited to silence inflammation and repair the injured and degenerating brain and spinal cord in both relapsing-remitting and progressive forms of MS and may be beneficial in other neurologic and psychiatric conditions.
\end{abstract}

It is difficult to say a favorable word about a terrible disease, but one of the positive features of multiple sclerosis (MS) is the remarkable capacity for patients to spontaneously recover from neurologic deficits that are attributed to inflammatory attacks on the CNS. About $80 \%$ of patients initially present with a decade or more of relapsing attacks in various areas of the CNS. Most of the neurologic deficits in these recurrent acute attacks, known as relapses after the initial episode, resolve nearly completely over a few days to a few weeks (1). For example, an attack on the optic nerve can leave an individual unable to read for a few days or weeks, but in many cases, there is full recovery of visual acuity after the initial neurologic insult. An attack within the pyramidal system in the brain or spinal cord may cause paralysis of a limb, but often there is recovery from this deficit over days and weeks in the early stages of MS. Despite these remissions, the course of disease advances over a decade or more in about a quarter of individuals. In these unfortunate individuals, relapsing-remitting disease transitions to secondary progressive MS, which is characterized by a large burden of disability and a lack of distinct relapses (1).

The substantial and remarkable recovery (remission) from an inflammatory insult (relapse) is a well-known phenomenon; however, more attention has been given to analyzing the inflammation that produces clinical deficits than to the processes that account for remission. The brain in MS frequently responds to immune damage with an array of molecules that serve to protect it from further damage and to foster recovery. This beneficial response to injury may be coordinated. Both cells extrinsic and intrinsic to the CNS are involved in the production of these guardian molecules. Some guardian molecules enter the somewhat privileged site of the brain via infiltrating

Conflict of interest: The author receives consulting income from Teva Pharmaceutical Industries Ltd., Raptor Pharmaceuticals, Atreca Inc., and Tolerion Inc. Research support was provided by Biogen Idec, Pfizer, and Japan Tobacco Central Pharmaceutical Research Institute.

Reference information: J Clin Invest. 2015;125(4):1371-1378. doi:10.1172/JCI74255. immune cells (1-6), still others are present at the blood-brain barrier that forms an interface between the immune system and the brain (7-11), while others are produced within the CNS itself (12-35).

This Review will describe these guardian molecules including protective cytokines like type 1 interferon, IL-10, and IL-27; the neurotrophins; neurotransmitters like GABA; antioxidants; small lipids present in the normal myelin sheath; nuclear hormone receptors; amyloid-forming molecules; and serpins and other inhibitory proteins. All these molecules may serve as platforms for novel restorative therapies, provided they can be delivered to the brain structures under attack.

\section{Protective cytokines: IL-10 and IL-27}

The immune attack in MS is driven primarily by the migration of lymphoid cells from outside the brain. T cells, B cells, and macrophages must move across the inflamed endothelium into brain via venules at the blood-brain barrier. The nature of this external attack on the CNS is best appreciated from studies leading to the development of the FDA-approved drug natalizumab (Figure 1), which is widely regarded as the most potent therapeutic to date for relapsing-remitting MS (RRMS). Natalizumab acts via binding to the $\alpha 4$ integrin, thereby impeding the migration of T and B cells and macrophages into the CNS. Preclinical studies first confirmed how blockade of $\alpha 4$ integrin reduced inflammatory cells in lesions in experimental autoimmune encephalomyelitis (EAE) (1,36-40). This therapy is associated with a marked reduction in inflammatory lesions, as measured by MRI.

The lymphocytes that migrate into the CNS produce proinflammatory mediators, including IFN- $\gamma$ and IL-17, which play fundamental roles in disease pathophysiology. Transcriptomic analysis and immunohistochemical staining of MS lesion material showed that IFN- $\gamma$ and IL-17 are prominent (41-43), and classic studies performed a quarter century ago demonstrated that administration of IFN- $\gamma$ worsened MS (44-46). However, immune cells migrating into the brain also produce cytokines like IL-10 
A

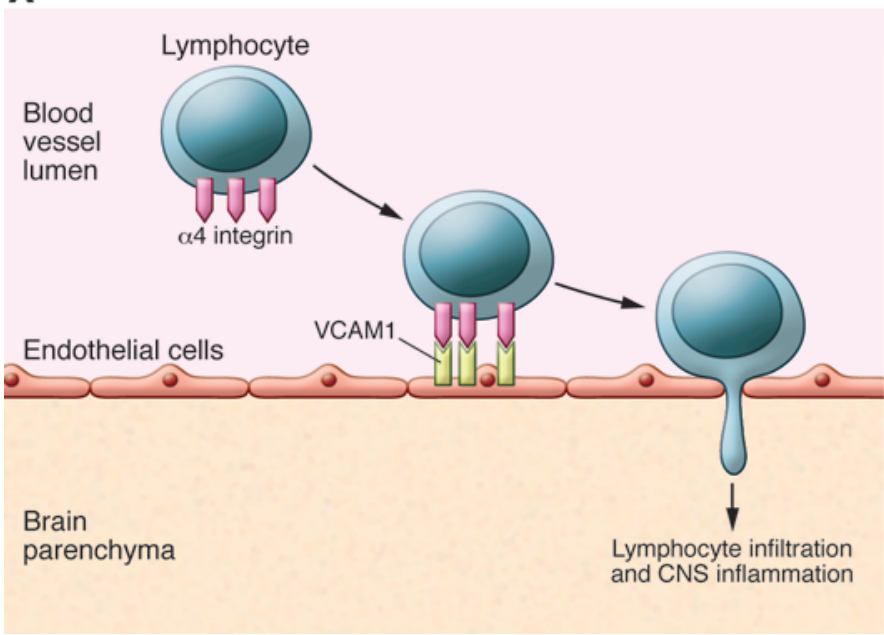

B

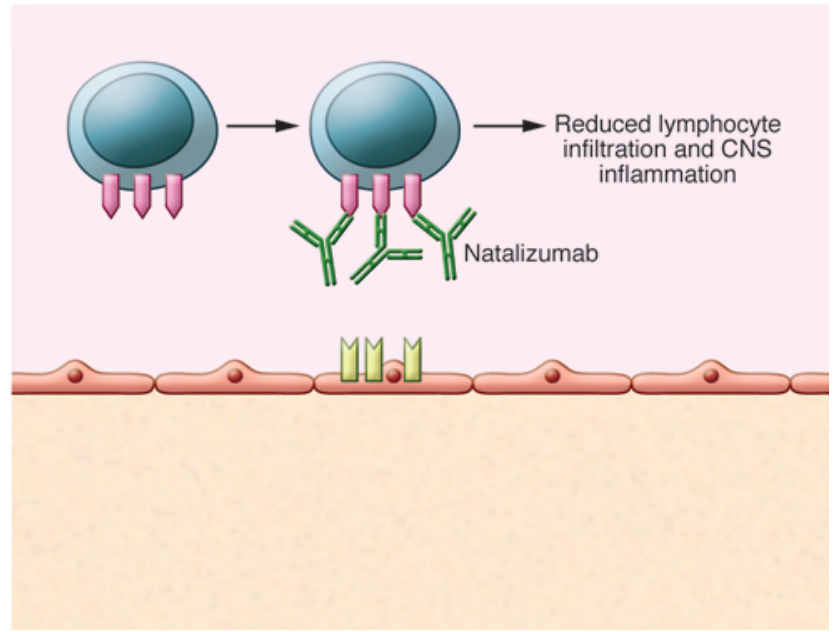

Figure 1. Natalizumab blocks lymphocyte homing in MS. (A) $\alpha 4$ integrin binds to vascular cell adhesion molecule 1 (VCAM1) on inflamed brain endothelium. This interaction gives lymphocytes access to the CNS. The presence of immune cells in the brain is a prominent feature of MS. (B) Natalizumab, a humanized antibody against $\alpha 4$ integrin, blocks binding of lymphocytes to VCAM on inflamed brain endothelium, thereby preventing lymphocyte entry into the CNS. Reproduced with permission from ref. 36 (Used with permission: () 2012 Steinman. Journal of Cell Biology. 199:413-416. doi: 10.1083/jcb.201207175).

and IL-27, two cytokines with suppressive properties that attenuate inflammation $(5,47)$. Protective cytokines like IL-10 are also produced by resident glia or neurons in response to injury. Activated microglia produce IL-10, which in turn suppresses neuroinflammation. Astrocytes also produce TGF- $\beta$ in response to IL-10, which further dampens inflammation in the brain (48).

The infiltrating lymphoid cells themselves can be modulated via therapeutics like IFN- $\beta$ to produce cytokines that suppress inflammation. The infiltrating lymphocytes then carry these suppressive cytokines to the locus of disease within the CNS. For example, IFN- $\beta$ induces the production of IL-10 and IL-27 and is widely used to treat RRMS $(5,47)$. Moreover, the intrinsic ability of a patient to produce IL-10 and IL-27 is associated with a beneficial clinical response to IFN- $\beta$, which is characterized by a reduction in the relapse rate and decreased inflammation in the brain $(5,47$, $49,50)$. IFN- $\beta$ induces Th1 cells to produce IL-10 and IL-27 (5), though it also acts to silence Th17 cells, thereby reducing levels of IL-17 (2-4). This is an example of how secretion of guardian molecules from cells that are not normally in the CNS may be an unexpected beneficial consequence of the migration of lymphocytes, particularly since this migration includes immune cells that induce disease in the first place.

\section{Neurotrophins: brain-derived neurotrophic factor and nerve growth factor}

Neurotrophins, including brain-derived neurotrophic factor (BDNF) and nerve growth factor (NGF), have long been regarded as molecules that promote neural outgrowth, but they are also imbued with antiinflammatory properties. Neurotrophins are produced in the brain at the site of disease as well as by the lymphoid cells that migrate into the brain in MS $(20,21,51)$, where they contribute to resolution of neuroinflammation.

BDNF was found in MS lesions, where it is produced in infiltrating immune cells. BDNF in the lesion acts on neurons via the neuronal $\mathrm{BDNF}$ receptor TRKB to rescue neurons after injury $(20,21,52)$. Approved drugs for RRMS, such as glatiramer acetate, induce BDNF, which may account for their therapeutic action. Mice with BDNF-deficient astrocytes or immune cells have worsened paralysis in EAE, with greater pathology in axons compared with that seen in WT mice (52). Certain polymorphisms in BDNF, including Val66Met, are protective in individuals with MS and are associated with a slower decrease in brain volume as disease progresses $(53,54)$.

NGF may also have a beneficial role in MS. NGF can be detected in active MS lesions with mass spectroscopy (6) and by immunohistochemical techniques (55). The NGF receptor was present in active lesions on astrocytes and microglia, as well as on infiltrating lymphocytes (55). NGF administered directly into the ventricles of the brain attenuated $\mathrm{T}$ cell-mediated inflammation in a model of MS in the marmoset, changing cytokine production in the CNS from a proinflammatory Th1 response, characterized by IFN- $\gamma$, to an antiinflammatory Th2 response, typified by IL-10 production (56). Neurotrophins are not always beneficial, as activation of TRKB in astrocytes enhances neurodegeneration in EAE (57).

A pattern appears again in these dual properties of neurotrophin molecules, which exert both antiinflammatory and growth-promoting effects on neurons. Molecules imbued with these properties act to both inhibit inflammation and promote regrowth in the nervous system. The inescapable "molecular logic" is that unless inflammation is attenuated, growth and regeneration are likely to be impaired. Neurotrophins therefore act as a remarkable molecular "solution" to both problems at the site of disease. These observations also emphasize that the injured brain fights back to restore itself after injury.

\section{Neurotransmitters: GABA}

GABA is best known as the main inhibitory neurotransmitter in the CNS. Surprisingly, the cells of the immune system have the capacity to synthesize and degrade GABA and to express enzymes involved in GABA uptake and the receptors that mediate GABA 
A

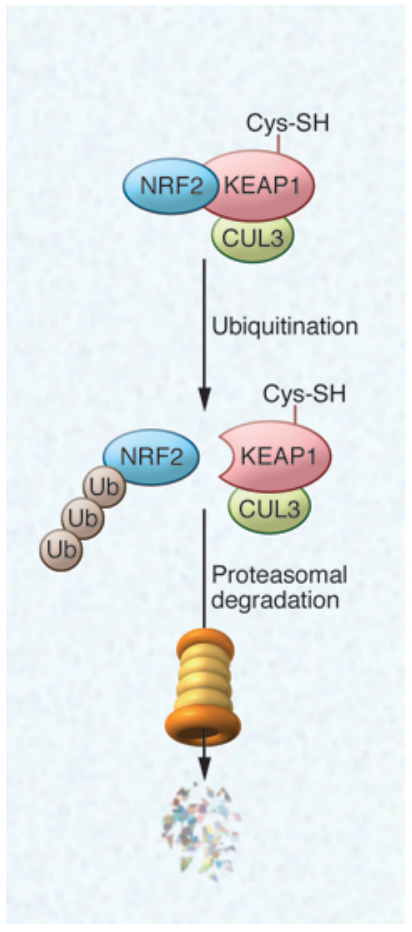

B

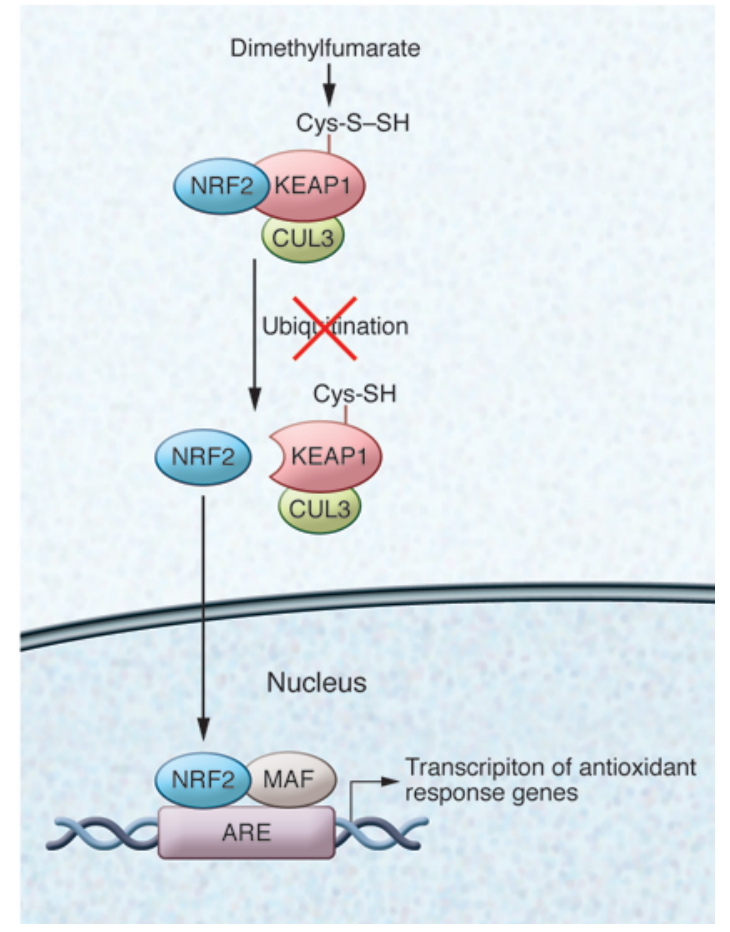

Figure 2. Mechanism of action of dimethylfumarate in the NRF2 pathway. Inflammation and oxidative stress are thought to promote tissue damage and MS, and recent data point to a protective role for antioxidant pathways, including the transcription factor NRF2, in MS. The drug dimethylfumarate, which targets the NRF2 pathway, is approved for treatment of RRMS and is under investigation in clinical trials for progressive forms of MS. Dimethylfumarate has pleiotropic mechanisms of action, but activation of NRF2 accounts for some of its antiinflammatory activity. (A) KEAP1, which is part of a cullin family E3 ubiquitin ligase complex, normally mediates ubiquitination and proteasomal degradation of NRF2. (B) When KEAP1 undergoes sulfhydration of cysteine 151 via binding of fumarate, it becomes unable to interact with NRF2, which accumulates in the nucleus and induces the expression of NRF2-dependent antioxidant and cytoprotective genes. Activation of NRF2 leads to increased production of a spectrum of antioxidants, including glutathione and cystathionine. signaling. Antigen-presenting cells (APCs) express functional GABA receptors and respond to GABA, as demonstrated by patchclamp electrophysiology. GABA itself and GABA agonists act directly on APCs, decreasing MAPK signaling and diminishing subsequent adaptive inflammatory responses to myelin proteins, including a reduction of Th1 and Th17 production (12).

Pharmacologic agents that activate GABA are able to modulate $\mathrm{T}$ cell function $(12,58)$ and reduce ongoing paralytic disease and brain inflammation in various animal models of EAE (12). The effect of GABA and drugs that increase GABA activity also show efficacy in models of other inflammatory diseases including inflammatory bowel disease (59), and in type 1 diabetes (60). Thus, GABA serves not only as an inhibitory neurotransmitter, but also exerts a parallel inhibitory effect on inflammation in the brain as well as on other organ systems. Clinical trials with pharmacological agents that affect GABA are planned.

\section{Antioxidants: glutathione and cystathionine}

Redox is implicated in the inflammatory pathology in general, and glutathione metabolites involved in glutathione metabolism, including glutathione synthase and various glutathione transferases, are expressed in active and chronic MS lesions (6). Molecules associated with maintaining a physiological redox state, including cystathione and glutathione, which are present in the brain, serve as regulators of the inflammatory response. A coordinated antioxidant response in MS is in part controlled by the transcription factor nuclear factor (erythroid-derived 2)-like 2(NRF2). Activation of NRF2 leads to increased production of a spectrum of antioxidants, including glutathione and cystathionine (61). NRF2 is activated upon its translocation to the nucleus, which is dependent on the modification of free cysteine residues, a process known as sulfhydration, in the NRF2-binding adaptor protein kelch-like ECH-associated protein (KEAP1).
The drug dimethylfumarate, which targets the NRF2 pathway, is approved for treatment of RRMS $(62,63)$ and is under investigation in clinical trials in progressive forms of MS. Dimethylfumarate has pleiotropic mechanisms of action, but activation of NRF2 accounts for some of its antiinflammatory activity (16, 17, 64). When KEAP1 undergoes sulfhydration of cysteine 151 via binding of fumarate, it becomes unable to interact with NRF2, which accumulates in the nucleus and induces the expression of NRF2-dependent antioxidant and cytoprotective genes. The antioxidant activities may support its use in neurodegenerative processes associated with progressive forms of MS. It is remarkable that a small molecule like dimethylfumarate can lead to activation of a single transcription factor with such potent activity on the inflammatory component of MS. But activation of multiple beneficial pathways with a single small molecule is seen repeatedly in biology and medicine.

Small molecules, including glutathione, cysteine, the neuroprotectant cystathionine, and the gaseous neurotransmitter $\mathrm{H}_{2} \mathrm{~S}$, play a key role in attenuating neurological injury. In experimental models of MS, elevation of cysteine via administration of cystamine or $\mathrm{N}$-acetylcysteine ameliorates paralysis in the EAE model $(18,19)$. This protective mechanism may also be applicable to other neurodegenerative processes including Huntington's disease (HD), spinocerebellar ataxia type 1, and dentatorubralpallidoluysian atrophy $(13,65-69)$. Recent reports show that there may be clinical benefit of cysteamine in treating $\operatorname{HD}(70,71)$. A defect in cystathionine $\gamma$-lyase (CSE) may in fact underlie neurodegeneration in HD and other disorders including Parkinson's and perhaps progressive MS, providing a rationale for why small molecules like cysteamine are effective $(13,61)$. Cysteamine also induces production of BDNF, which has both antiinflammatory and neurotrophic properties, as discussed above $(68,69)$. 
These small molecules also catalyze sulfhydration $(64,65,71)$. As described above, the interplay of sulfhydrates on KEAP1 has remarkable similarities to the mechanism of action of dimethylfumarate (Figure 2) in allowing the nuclear translocation of NRF2. Proteomic platforms allow us to analyze the various molecules that bind small-molecule mediators like dimethylfumarate and cysteamine (72). Such analysis will elucidate the parallel biochemical pathways that might be mediated in attenuating not only inflammation in the CNS, but the degenerative processes $(73,74)$.

\section{Protective gasotransmitters}

Gaseous neurotransmitters, also known as gasotransmitters $(13,14)$, like $\mathrm{H}_{2} \mathrm{~S}$ (involved in sulfhydration) and NO (involved in nitrosylation), are not the only gaseous protectants from neuroinflammation. Administration of $\mathrm{CO}$ reverses paralysis in EAE. The effect of CO is mediated by heme oxygenase- 1 (HMOX1). HMOX1 dampens inflammatory reactions via the catabolism of heme into $\mathrm{CO}$, iron, and biliverdin in the brain, liver, spleen, and endothelium $(75,76)$. The protective effect of HMOX1 is associated with inhibition of MHC class II expression by APCs and inhibition of $\mathrm{CD}^{+}{ }^{+}$Th and $\mathrm{CD} 8^{+}$ T cell accumulation. Activation of myelin-reactive $\mathrm{T}$ cells is mediated via HMOX1 lymphocyte proliferation and effector function within the CNS. Of interest, the NRF2 pathway is linked to HMOX1. The BACH1/MAFK transcriptional repressor complex blocks expression of $H M O X 1$. Binding of heme to BACH1 releases the complex from DNA, allowing NRF2-mediated transcription (77-80).

Enhancing levels of gaseous neurotransmitters is under serious consideration as a neuroprotective therapy for MS on the basis of its success in animal models, in which delivery of CO via inhalation of $\mathrm{CO}$, compared with room air, arrested EAE progression and paralysis (75).

\section{Fatty acids}

Naturally occurring lipids that comprise more than $70 \%$ of the myelin sheath are targeted by the adaptive immune system (15). Antibody and $\mathrm{T}$ cell responses to lipids are detected in both relapsingremitting and progressive forms of MS (15). We used lipid antigen microarrays and lipid mass spectrometry to identify the lipid targets that react with antimyelin antibodies in MS brain. We reported that autoantibodies in MS target a phosphate group in phosphatidylserine and oxidized phosphatidylcholine derivatives. Remarkably, when administered systemically, these same lipids that are targeted via antibodies actually ameliorated experimental autoimmune encephalomyelitis. The mechanism of action was via suppression of activation of autoreactive T cells as well as induction of apoptosis of autoreactive T cells (15). These effects were mediated by the lipids' saturated fatty acid side chains and did not require the entire phospholipid backbone. Thus, substituents of naturally occurring phospholipids represent a natural antiinflammatory class of compounds that have potential as therapeutics for MS (15). Other naturally occurring lipids are proinflammatory. Lactosylceramide activates microglia and astrocytes and contributes to neurodegenerative processes in experimental neuroinflammation (81).

\section{Nuclear hormone receptors}

Females are far more susceptible to MS than are males, and this ratio has been increasing over the past 50 years (1). In investigat- ing some of the factors controlling this dimorphism in susceptibility, we have encountered a potent brake on the immune system that may play an import role in neuroinflammation and neurodegeneration. The PPARs are a family of nuclear hormone-activated receptors that act as transcription factors. These receptors are activated by sex hormones, lipids, and fatty acids (22-24).

In the EAE model, PPAR $\alpha$ is expressed on T cells at a higher level in males than in females. Females are far more susceptible to disease (82). Lovett-Racke and we showed independently that PPAR $\alpha$ agonists attenuate paralysis and inflammation in EAE (23, 83). Castration of males also lowers PPAR $\alpha$ expression and renders males more susceptible to EAE (82). Conversely, higher levels of PPAR $\alpha$ are expressed when females are given testosterone (22). Dunn showed that in EAE, females had a stronger Th1 response driven by IFN- $\gamma$ than did males (82) and that this sexual dimorphism in the Th1 response was regulated by PPAR $\alpha(22,82)$.

In 2012, Dunn and we translated these studies to humans (22). Female humans exhibit much stronger Th1 responses than do males, while males exhibit stronger Th17 responses than do females. In concordance with the studies in mice, androgens enhanced PPAR $\alpha$ expression in humans. Additionally, we demonstrated that PPAR $\gamma$ regulates Th17 responses in humans. PPAR $\gamma$ was shown to regulate and attenuate inflammatory reactions between myeloid cells and resident cells in the brain in neuroinflammation (83). One of the major implications of these studies is that androgen-driven transcription factors serve as a major braking system on both Th1 and Th17 T cell responses (22, 82). This braking system is operative in the peripheral immune system, but controls the expression of inflammation in the brain in EAE.

\section{Amyloid-forming molecules}

One of the most surprising developments in deciphering which molecules may serve as guardians in MS arose from an analysis of molecules found in MS lesions $(84,85)$. In 2002, we published a report on the transcriptome of acute and chronic lesions in MS $(41,86)$. Two years later, working with the same blocks of tissue, we performed proteomic analysis of laser-captured, microdissected lesions (6). We found molecules in MS lesions that included some of the more infamous amyloid molecules associated with pathology in Alzheimer's disease(AD) (A $\beta$ and tau), as well as the prion protein $\operatorname{PrP}^{\mathrm{c}}$. Abundant amounts of $\alpha \mathrm{B}$ crystallin (CRYAB), another amyloid molecule, were also found in MS lesions, as we had already seen by transcriptional profiling $(41,86)$ as well as by immunohistochemical studies $(35,87)$. We noted that several amyloid-forming molecules, including CRYAB, amyloid precursor protein (APP), major prion protein, and tau, were all found in lesions that were laser captured, microdissected, and subjected to mass spectral analysis (6).

Most of us continue to think of amyloid-forming molecules as harmful. In the community of scientists studying AD, the amyloid-forming molecules tau and $A \beta$ are considered central players in its pathophysiology (88). A large body of literature exists describing the proinflammatory properties of amyloid-forming proteins, particularly $\mathrm{A} \beta$; yet, the pathology of $\mathrm{AD}$ shows little evidence of inflammation and no evidence of classic innate or adaptive immunity and inflammation in lesions $(74,89)$, a situation that is vastly different from that seen in MS. In MS, there is a 
significant imprint of inflammation with perivascular infiltrates, with evidence of both innate immunity and adaptive immunity at the $\mathrm{T}$ and $\mathrm{B}$ cell levels (89). To refer to neuroinflammation in $\mathrm{AD}$ is perhaps to give new meaning to the word inflammation. We make these transformations in language all the time, and this may be an example in the realm of biomedical science. Consider that in the realm of finance, when we use the word "credit," we mean that we are assuming "debt" (90). Are we fooling ourselves when we apply the word "inflammation" to the pathology in AD, in which the hallmarks of inflammation are largely absent? To develop novel therapies for $\mathrm{AD}$, the pharmaceutical industry has made huge investments in an attempt to lower $A \beta$ by inhibiting the secretase enzymes involved in $A \beta$ formation, or by administering vaccines or monoclonal antibodies to somehow remove these molecules from the brain. Numerous phase 3 trials using these strategies have failed $(90,91)$. Could amyloid molecules have a different role in MS $(30,31)$ ? Research on MS and its animal models point in this direction.

CRYAB is a classic example of an amyloid-forming molecule that forms $\beta$ zippers, incorporates thioflavin T, and aggregates into fibrils. A role for CRYAB in the pathogenesis of MS was first discovered in 1995 (87), when Van Noort and colleagues observed that CRYAB was the most immunogenic component of the myelin sheath for the T cells in MS patients (87). In 2001, we noted that CRYAB was the most abundant transcript found in MS lesions, but not in healthy brains (84).

In a series of experiments testing the administration of CRYAB in several preclinical models of disease, including EAE, we discovered that i.v. administration of recombinant CRYAB reversed paralysis and attenuated inflammation. The effects were robust in several versions of EAE, including in models of progressive disease and relapsing-remitting disease. CRYAB was also efficacious in models of stroke, myocardial infarction, optic nerve ischemia, and rheumatoid arthritis (92-96). Investigations in optic nerve ischemia showed that administration of CRYAB after disease onset induced complete rescue of the optic nerve oligodendrocytes from the ischemic insult (93). These experimental results suggest that amyloid-forming proteins, which are found in actual lesions in MS, may serve to contain ongoing inflammation and initiate repair. Among the various amyloidforming molecules, the case is strongest for CRYAB in protecting and reversing neuroinflammation and in protecting myelinforming oligodendrocytes.

While we have shown in gain-of-function experiments that administration of amyloid-forming proteins such as CRYAB improves function in EAE, stroke, myocardial infarction, and optic nerve ischemia (92-96), loss-of-function experiments with genetic deletion of amyloid-forming proteins provide further support for the notion that amyloid proteins and peptides are beneficial. It is noteworthy that clinical signs and inflammation in EAE are exacerbated in mice lacking CRYAB (35), APP (30), the major prion protein PRP (33), serum amyloid P (34), and tau (32) compared with what is seen in WT animals. As mentioned, APP, tau, and CRYAB are all found in MS lesions (6), indicating that their presence may indeed attenuate, rather than exacerbate, inflammation in MS. Mice lacking APP also have more severe functional disturbances following blunt brain trauma (30).
Taken together, the gain-of-function and loss-of-function experiments indicate that amyloid molecules forming self-assembling, zippered nanostructures are potent mediators of protection and repair. This is a stunning reversal of the conventional perspective for these molecules. The conclusion of these experiments to date is that CRYAB, as well as other amyloid-forming molecules, may play a guardian role in MS.

Having observed the guardian properties of CRYAB in various models of neuroinflammation as well as inflammation outside the brain and in various ischemic models, we asked whether there was a core structure common to CRYAB and other amyloid-forming molecules. Eisenberg (88) identified a common hexapeptide core motif found in many amyloids, including $\mathrm{A} \beta \mathrm{A} 4$, tau, amylin, prion proteins, serum amyloid protein $\mathrm{P}$, and CRYAB. We explored whether these hexapeptides with a propensity to form self-assembling zippered $\beta$ sheets might be therapeutic.

The core hexapeptide structure is highly immunosuppressive and can reverse paralysis in EAE when administered systemically. The hexapeptides bind a set of proinflammatory mediators in plasma, including acute-phase reactants and complement components. Administration of this amyloid-forming hexapeptide quickly lowers inflammatory cytokines in plasma such as IL-2 and IL-6, which are highly expressed in both acute and chronic MS lesions $(31,41,86)$. The beneficial properties of amyloid-forming hexapeptides provide a potential new therapeutic direction. These experiments indicate that amyloid-forming molecules have Janus faces, providing unexpected benefit for neuroinflammatory conditions.

\section{Serpins and other inhibitory proteins}

A number of inhibitory proteins were detected at the site of MS lesions using transcriptional gene profiling and proteomics $(6,44)$. Notably, the complement mediators C1R and C3 are found in MS lesions; complement damage plays a major role in demyelination. A soluble inhibitor of complement activation, sCRRY, reverses EAE (26). It is therefore noteworthy that the inflamed brain in MS produces its own endogenous inhibitor of complement, CD59, also known as protectin or membrane attack complex inhibitory protein $(25,44)$. Membrane attack complexes are also found in the spinal fluid of MS patients (97). Other inhibitors of proteases associated with inflammation have been detected in MS brain, including serpins like $\alpha 1$ antichymotrypsin (41). Sustained expression of $\alpha 1$ antichymotrypsin ameliorated EAE and enhanced expression of Tregs (98).

A study of laser-captured, microdissected MS lesions provided evidence of activation of the coagulation cascade, including tissue factor and protein $\mathrm{C}$ inhibitor, particularly in chronic MS lesions. Administration of activated protein $\mathrm{C}$ ameliorated $\mathrm{EAE}$ and suppressed Th1 and Th17 cytokines in astrocytes and immune cells (6). Activated protein $\mathrm{C}$ signals through protease-activated receptor 1 (PAR-1) and endothelial protein $\mathrm{C}$ receptor (EPCR). This again emphasizes that control of chronic degenerative processes may be regulated at the blood-brain barrier interface. It may not be strictly necessary to develop compounds that actually penetrate the blood-brain barrier. An activated protein $\mathrm{C}$ analog stimulated neuron production from human neural progenitor cells via modulation of a pathway involving PAR/PAR-3/sphingosine-1phosphate receptor $1 / \mathrm{AKT}$ (99). Other components of the coagu- 
lation cascade are upregulated in acute MS (6). Inhibition of the thrombin pathway, which is activated in acute MS lesions, is also effective in ameliorating paralytic disease. Treatment with the anticoagulant hirudin induced dramatic improvement in disease severity, with suppression of the cytokines IL-6, TNF, and IL-17 (6). Our understanding of guardian molecules must now expand to include components of the complement and clotting cascades, elements of which protect against neuropathology.

\section{Coda}

There is a vast array of molecules that have protective properties in MS lesions. Identification of these molecules and their targets may lead us to new therapies for protection and repair in MS. Repair and protection may not be independent of the suppression of unwanted inflammation, and some targets may help to ameliorate the entire constellation of pathology, inhibiting autoimmunity and promoting protection and regeneration simultaneously. As we teach medical science and as we continue to learn about new developments, it is increasingly clear that the segregation of physiologic systems is actually untenable. As we can see, molecules like $\mathrm{CO}$ that have famously been associated with death from asphyxia can be harnessed to reverse paralysis and neuroinflammation. Molecules associated with the coagulation cascade play important roles in attenuation of inflammation. Molecules not only have their good face and their bad face - the Janus concept but are likely to defy convenient characterization. Surprises abound as we come to understand the role of molecules in different pathological contexts.

\section{Acknowledgments}

The author wishes to acknowledge the National Multiple Sclerosis Society and the NIH for funding his work over the past 35 years and his patients with MS, whose courage inspires him.

Address correspondence to: Lawrence Steinman, Beckman Center for Molecular Medicine, RM B002, Stanford University, Stanford, California 94350, USA. Phone: 650.725.6401; E-mail: steinman@stanford.edu.
1. Steinman L. Immunology of relapse and remission in multiple sclerosis. Annu Rev Immunol. 2014;32:257-281.

2. Fitzgerald DC, et al. Suppression of autoimmune inflammation of the central nervous system by interleukin 10 secreted by interleukin 27-stimulated T cells. Nat Immunol. 2007; 8(12):1372-1379.

3. Fitzgerald DC, et al. Independent and interdependent immunoregulatory effects of IL-27, IFN- $\beta$, and IL-10 in the suppression of human Th17 cells and murine experimental autoimmune encephalomyelitis. JImmunol. 2013;190(7):3225-3234.

4. Murugaiyan G, Mittal A, Weiner HL. Identification of an IL-27/osteopontin axis in dendritic cells and its modulation by IFN- $\gamma$ limits IL-17mediated autoimmune inflammation. Proc Natl Acad Sci U S A. 2010;107(25):11495-11500.

5. Axtell RC, et al. T helper type 1 and 17 cells determine efficacy of IFN- $\beta$ in multiple sclerosis and experimental encephalomyelitis. Nature Med. 2010;16(4):406-412.

6. Han MH, et al. Proteomic analysis of active multiple sclerosis lesions reveals therapeutic targets. Nature. 2008;451(7182):1076-1081.

7. Brinkmann V, et al. Fingolimod (FTY720): discovery and development of an oral drug to treat multiple sclerosis. Nat Rev Drug Discov. 2010;9(11):883-897.

8. Choi JW, et al. FTY720 (fingolimod) efficacy in an animal model of multiple sclerosis requires astrocyte sphingosine 1-phosphate receptor 1 (S1P1) modulation. Proc Natl Acad Sci U S A. 2011;108(2):751-756.

9. Calabresi PA, et al. Safety and efficacy of fingolimod in patients with relapsing-remitting multiple sclerosis (FREEDOMS II): a double-blind, randomised, placebo-controlled, phase 3 trial. Lancet Neurol. 2014;13(6):545-556.

10. Khatri B, et al. Comparison of fingolimod with interferon beta-1a in relapsing-remitting multiple sclerosis: a randomised extension of the TRANSFORMS study. Lancet Neurol. 2011;10(6):520-529.

11. Czech B, et al. The immunomodulatory sphin- gosine 1-phosphate analog FTY720 reduces lesion size and improves neurological outcome in a mouse model of cerebral ischemia. Biochem Biophys Res Commun. 2009;389(2):251-256.

12. Bhat $\mathrm{R}$, et al. Inhibitory role for GABA in autoimmune inflammation. Proc Natl Acad Sci US A. 2010;107(6):2580-2585.

13. Paul BD, et al. Cystathionine $\gamma$-lyase deficiency mediates neurodegeneration in Huntington's disease. Nature. 2014;509(7498):96-101.

14. Morikawa T, et al. Hypoxic regulation of the cerebral microcirculation is mediated by a carbon monoxide-sensitive hydrogen sulfide pathway. Proc Natl Acad Sci U S A. 2012;109(4):1293-1298.

15. Ho PP, et al. Identification of naturally occurring fatty acids of the myelin sheath that resolve neuroinflammation. Sci Transl Med. 2012;4(137):137ra73.

16. Linker RA, et al. Fumaric acid esters exert neuroprotective effects in neuroinflammation via activation of the Nrf2 antioxidant pathway. Brain. 2011;134(pt 3):678-692.

17. Ghoreschi K, et al. Fumarates improve psoriasis and multiple sclerosis by inducing type II dendritic cells. J Exp Med. 2011;208(11):2291-2303.

18. Lehmann D, Karussis D, Misrachi-Koll R, Shezen E, Ovadia H, Abramsky O. Oral administration of the oxidant-scavenger N-acetyl-L-cysteine inhibits acute experimental autoimmune encephalomyelitis. J Neuroimmunol. 1994;50(1):35-42.

19. Oh K, Park HB, Seo MW, Byoun OJ, Lee DS. Transglutaminase 2 exacerbates experimental autoimmune encephalomyelitis through positive regulation of encephalitogenic $\mathrm{T}$ cell differentiation and inflammation. Clin Immunol. 2012;145(2):122-132.

20. Stadelmann C, et al. BDNF and gp145trkB in multiple sclerosis brain lesions: neuroprotective interactions between immune and neuronal cells? Brain. 2002;125(pt 1):75-85.

21. Kerschensteiner M, et al. Activated human T cells, $B$ cells, and monocytes produce brain-derived neurotrophic factor in vitro and in inflammatory brain lesions: a neuroprotective role of inflammation? J Exp Med. 1999;189(5):865-870.

22. Zhang MA, et al. Peroxisome proliferatoractivated receptors (PPAR) $-\alpha$ and $-\gamma$ regulate IFN $\gamma$ and IL-17A production by human T cells in a sex-specific way. Proc Natl Acad Sci US A. 2012;109(24):9505-9510.

23. Lovett Racke AE, et al. Peroxisome proliferator-activated receptor alpha agonists as therapy for autoimmune disease. JImmunol. 2004;172(9):5790-5798.

24. Mukundan L, et al. PPAR- $\Delta$ senses and orchestrates clearance of apoptotic cells to promote tolerance. Nature. 2009;15(11):1266-1272.

25. Walsh LA, Tone M, Thiru S, Waldmann H. The CD59 antigen - a multifunctional molecule. Tissue Antigens. 1992;40(5):213-220.

26. Davoust N, Nataf S, Reiman R, Holers MV, Campbell IL, Barnum SR. Central nervous system-targeted expression of the complement inhibitor sCrry prevents experimental allergic encephalomyelitis. J Immunol. 1999;163(12):6551-6556.

27. Esmon CT. Crosstalk between inflammation and thrombosis. Maturitas. 2004;47(4):305-314

28. Eddleston M, de la Torre JC, Oldstone MB, Loskutoff DJ, Edgington TS, Mackman N. Astrocytes are the primary source of tissue factor in the murine central nervous system. A role for astrocytes in cerebral hemostasis. J Clin Invest. 1993;92(1):349-358.

29. Gerlitz B, Grinnell BW. Mutation of protease domain residues Lys37-39 in human protein C inhibits activation by the thrombomodulin-thrombin complex without affecting activation by free thrombin. J Biol Chem. 1996;271(37):22285-22288.

30. Grant JL, et al. Reversal of paralysis and reduced inflammation from peripheral administration of $\beta$-amyloid in TH1 and TH17 versions of experimental autoimmune encephalomyelitis. Sci Transl Med. 2012;4(145):145ra105.

31. Kurnellas MP, Adams CM, Sobel RA, Steinman L, 
Rothbard JR. Amyloid fibrils composed of hexameric peptides attenuate neuroinflammation. Sci Transl Med. 2013;5(179):179ra42.

32. Weinger JG, et al. Mice devoid of Tau have increased susceptibility to neuronal damage in myelin oligodendrocyteglycoprotein-induced experimental autoimmune encephalomyelitis. J Neuropathol Exp Neurol. 2012;71(5):422-433.

33. Gourdain P, Ballerini C, Nicot AB, Carnaud C. Exacerbation of experimental autoimmune encephalomyelitis in prion protein (PrPc)-null mice: evidence for a critical role of the central nervous system. J Neuroinflammation. 2012;9:25.

34. Ji Z, Ke ZJ, Geng JG. SAP suppresses the development of experimental autoimmune encephalomyelitis in C57BL/6 mice. Immunol Cell Biol. 2012;90(4):388-395.

35. Ousman SS, et al. Protective and therapeutic role for alphaB-crystallin in autoimmune demyelination. Nature. 2007;448(7152):474-479.

36. Steinman L. The discovery of natalizumab, a potent therapeutic for multiple sclerosis. J Cell Biol. 2012;199(3):413-416.

37. Yednock TA, Cannon C, Fritz LC, SanchezMadrid F, Steinman L, Karin N. Prevention of experimental autoimmune encephalomyelitis by antibodies against $\alpha 4 \beta 1$ integrin. Nature. 1992;356(6364):63-66.

38. Polman $\mathrm{CH}$, et al. A randomized, placebo-controlled trial of natalizumab for relapsing multiple sclerosis. N Engl J Med. 2006;354(9):899-910.

39. Rudick RA, et al. Natalizumab plus interferon $\beta$-1a for relapsing multiple sclerosis. $\mathrm{N} \mathrm{Engl} \mathrm{J} \mathrm{Med.}$ 2006;354(9):911-923.

40. Rudick R, Polman C, Clifford D, Miller D, Steinman L. Natalizumab: bench to bedside and beyond. JAMA Neurol. 2013;70(2):172-182.

41. Lock C, et al. Gene-microarray analysis of multiple sclerosis lesions yields new targets validated in autoimmune encephalomyelitis. Nat Med. 2002;8(5):500-508.

42. Tzartos JS, et al. Interleukin-17 production in central nervous system-infiltrating $T$ cells and glial cells is associated with active disease in multiple sclerosis. Am J Pathol. 2008;172(1):146-155.

43. Matusevicius D, et al. Interleukin-17 mRNA expression in blood and CSF mononuclear cells is augmented in multiple sclerosis. Mult Scler. 1999;5(2):101-104.

44. Panitch HS, Hirsch RL, Haley AS, Johnson KP. Exacerbations of multiple sclerosis in patients treated with gamma interferon. Lancet. 1987;329(8538):893-895.

45. Panitch HS, Hirsch RL, Schindler J, Johnson KP. Treatment of multiple sclerosis with gamma interferon: exacerbations associated with activation of the immune system. Neurology. 1987;37(7):1097-1102.

46. Steinman L. A brief history of T(H)17, the first major revision in the $\mathrm{T}(\mathrm{H}) 1 / \mathrm{T}(\mathrm{H}) 2$ hypothesis of T cell-mediated tissue damage. Nat Med. 2007;13(2):139-145.

47. Sweeney CM, et al. IL-27 mediates the response to IFN- $\beta$ therapy in multiple sclerosis patients by inhibiting Th17 cells. Brain Behav Immun. 2011;25(6):1170-1181.

48. Norden DM, Fenn AM, Dugan A, Godbout JP. TGFB produced by IL-10 redirected astrocytes attenuates microglial activation. Glia. 2014;62(6):881-895.

49. Guo B, Chang EY, Cheng G. The type I IFN induction pathway constrains Th17-mediated autoimmune inflammation in mice. J Clin Invest. 2008;118(5):1680-1690.

50. Shinohara M, Kim JH, Garcia VA, Cantor H. Engagement of the type I interferon receptor on dendritic cells inhibits $\mathrm{T}$ helper 17 cell development: role of intracellular osteopontin. Immunity. 2008;29(1):68-78.

51. Linker RA, et al. CNTF is a major protective factor in demyelinating CNS disease: a neurotrophic cytokine as modulator in neuroinflammation. Nat Med. 2002;8(6):620-624.

52. Linker RA, et al. Functional role of brain-derived neurotrophic factor in neuroprotective autoimmunity: therapeutic implications in a model of multiple sclerosis. Brain. 2010;133(pt 8):2248-2263.

53. Zivadinov R, et al. Preservation of gray matter volume in multiple sclerosis patients with the Met allele of the rs6265 (Val66Met) SNP of brain-derived neurotrophic factor. $\mathrm{Hum} \mathrm{Mol}$ Genet. 2007;16(22):2659-2668.

54. Friese M, Schattling B, Fugger L. Mechanisms of neurodegeneration and axonal dystfunction in multiple sclerosis. Nat Rev Neurol. 2014;10(4):225-238.

55. Valdo P, et al. Enhanced expression of NGF receptors in multiple sclerosis lesions. J Neuropathol Exp Neurol. 2002;61(1):91-98.

56. Villoslada P, et al. Human nerve growth factor protects common marmosets against autoimmune encephalomyelitis by switching the balance of $\mathrm{T}$ helper cell type 1 and 2 cytokines within the central nervous system. J Exp Med. 2000;191(10):1799-1806

57. Colombo E, et al. Stimulation of the neurotrophin receptor TrkB on astrocytes drives nitric oxide production and neurodegeneration. JExp Med. 2012;209(3):521-535.

58. Tian J, Chau C, Hales TG, Kaufman DL. GABA(A) receptors mediate inhibition of $\mathrm{T}$ cell responses. J Neuroimmunol. 1999;96(1):21-28.

59. Diop L, Raymond F, Fargeau H, Petoux F. Chovet M, Doherty AM. Pregabalin (CI-1008) inhibits the trintorbenzene sulfonic acid-induced chronic colonic allodynia in the rat. J Pharmacol Exp Ther. 2002;302(3):1013-1022.

60. Tian J, Lu Y, Zhang H, Chau CH, Dang HN, Kaufman DL. Gamma-aminobutyric acid inhibits $\mathrm{T}$ cell autoimmunity and the development of inflammatory responses in a mouse type 1 diabetes model. J Immunol. 2004;173(8):5298-5304.

61. Yang $\mathrm{G}$, et al. Hydrogen sulfide protects against cellular senescence via S-sulfhydration of Keap1 and activation of Nrf2. Antioxid Redox Signal. 2013;18(15):1906-1919.

62. Gold R, et al. Placebo-controlled phase 3 study of oral BG-12 for relapsing multiple sclerosis. $N$ Engl JMed. 2012;367(12):1098-1107.

63. Fox R, et al. Placebo-controlled phase 3 study of oral BG-12 or glatiramer in multiple sclerosis. N Engl J Med. 2012;367(12):1087-1097.

64. Scannevin RH, et al. Fumarates promote cytoprotection of central nervous system cells against oxidative stress via the nuclear factor (erythroid-derived 2)-like 2 pathway. J Pharmacol Exp Ther. 2012;341(1):274-284.
65. Yang $\mathrm{G}$, et al. $\mathrm{H} 2 \mathrm{~S}$ as a physiologic vasorelaxant: hypertension in mice with deletion of cystathionine $\gamma$-lyase. Science. 2008;322(5901):587-590.

66. Karpuj MV, et al. Prolonged survival and decreased abnormal movements in transgenic model of Huntington disease, with administration of the transglutaminase inhibitor cystamine. Nat Med. 2002;8(2):143-149.

67. Igarashi S, et al. Suppression of aggregate formation and apoptosis by transglutaminase inhibitors in cells expressing truncated DRPLA protein with an expanded polyglutamine stretch. Nat Genet. 1998;18(2):111-117.

68. Borrell-Pagès $\mathrm{M}$, et al. Cystamine and cysteamine increase brain levels of BDNF in Huntington disease via HSJ1b and transglutaminase. JClin Invest. 2006;116(5):1410-1424.

69. Sun L, Xu S, Zhou M, Wang C, Wu Y, Chan P. Effects of cysteamine on MPTP-induced dopaminergic neurodegeneration in mice. Brain Res. 2010;1335:74-82.

70. Raptor Announces Clinical Results With RP103 in Huntington's Disease Phase 2/3 Trial [press release]. Novato, California, USA: Globe Newswire; February 20, 2014.

71. Prundean A, Youssov K, Humbert S, Bonneau D, Verny C. A phase II, open-label evaluation of cysteamine tolerability in patients with Huntington's disease. Mov Disord. 2015;30(2):288-289.

72. Lanning BR, et al. A road map to evaluate the proteome-wide selectivity of covalent kinase inhibitors. Nat Chem Biol. 2014;10(9):760-768.

73. Graeber MB. Neuroinflammation: no rose by any other name. Brain Pathol. 2014;24(6):620-622.

74. Steinman L. Why are prions and amyloid structures immune suppressive and other intriguing questions facing neuroimmunologists in the future. Rev Neurol (Paris). 2014;170(10):602-607.

75. Chora AA, et al. Heme oxygenase-1 and carbon monoxide suppress autoimmune neuroinflammation. JClin Invest. 2007;117(2):438-447.

76. Motterlini R, Otterbein LE. The therapeutic potential of carbon monoxide. Nat Rev Drug Discov. 2010;9(9):728-743.

77. Wilson AJ, Kerns JK, Callahan JF, Moody CJ. Keap calm, and carry on covalently. J Med Chem. 2013;56(19):7463-7476.

78. Sun J, et al. Hemoprotein Bach1 regulates enhancer availability of heme oxygenase-1 gene. $E M B O J$. 2002;21(19):5216-5224.

79. Reichard JF, Motz GT, Puga A. Heme oxygenase-1 induction by NRF2 requires inactivation of the transcriptional repressor BACH1. Nucleic Acids Res. 2007;35(21):7074-7086.

80. Pamplona A, et al. Heme oxygenase-1 and carbon monoxide suppress the pathogenesis of experimental cerebral malaria. Nat Med. 2007;13(6):703-710.

81. Mayo L, et al. Regulation of astrocyte activation by glycolipids drives chronic CNS inflammation. Nat Med.2014;20(10):1147-1156.

82. Dunn SE, et al. Peroxisome proliferator-activated receptor (PPAR) $\alpha$ expression in $\mathrm{T}$ cells mediates gender differences in development of T cell-mediated autoimmunity. J Exp Med. 2007;204(2):321-330.

83. Hucke S, et al. Licensing of myeloid cells promotes central nervous system autoimmunity and 
is controlled by peroxisome proliferator-activated receptor $\gamma$. Brain. 2012;135(pt 5):1586-1605.

84. Chabas D, et al. The influence of the pro-inflammatory cytokine, osteopontin, on autoimmune demyelinating disease. Science. 2001;294(5547):1731-1735.

85. Steinman L, Zamvil S. Transcriptional analysis of targets in multiple sclerosis. Nat Rev Immunol. 2003;3(6):483-492.

86. Zamvil SS, Steinman L. Diverse targets for intervention during inflammatory and neurodegenerative phases of multiple sclerosis. Neuron. 2003;38(5):685-688.

87. van Noort JM, et al. The small heat-shock protein alpha B-crystallin as candidate autoantigen in multiple sclerosis. Nature. 1995;375(6534):798-801.

88. Laganowsky A, et al. Atomic view of a toxic amyloid small oligomer. Science. 2012;335(6073):1228-1231.

89. Steinman L. Nuanced roles of cytokines in three major human brain disorders. J Clin Invest. 2008;118(11):3557-3563.
90. Karran E, Hardy J. A critique of the drug discovery and phase 3 clinical programs targeting the amyloid hypothesis for Alzheimer disease. Ann Neurol. 2014;76(2):185-205.

91. Hyman BT, Sorger P. Failure analysis of clinical trials to test the amyloid hypothesis. Ann Neurol. 2014;76(2):159-161.

92. Steinman L, Ousman SS, Robinson WH, inventors; The Board Of Trustees Of The Leland Stanford Junior University, assignee. Alpha B-crystallin as a therapy for rheumatoid arthritis. US patent 7,875,589. December 11, 2007.

93. Steinman L, Ousman SS, Robinson WH, inventors; The Board Of Trustees Of The Leland Stanford Junior University, assignee. Alpha B-crystallin as a therapy for ischemia or inflammation. US patent 8,771,689. June 23, 2011.

94. Pangratz-Fuehrer S, Kaur K, Ousman SS, Steinman L, Liao YJ. Functional rescue of experimental ischemic optic neuropathy with $\alpha \mathrm{B}$-crystallin. Eye (Lond). 2011;25(6):809-817.

95. Velotta JB, et al. $\alpha B$-crystallin improves murine car- diac function and attenuates apoptosis in human endothelial cells exposed to ischemia-reperfusion. Ann Thorac Surg. 2011;91(6):1907-1913.

96. Arac A, et al. Systemic augmentation of alphaB-crystallin provides therapeutic benefit twelve hours post-stroke onset via immune modulation. Proc Natl Acad Sci U S A. 2011;108(32):13287-13292.

97. Scolding NJ, Morgan BP, Houston WA, Linington C, Campbell AK, Compston DA. Vesicular removal by oligodendrocytes of membrane attack complexes formed by activated complement. Nature. 1989;339(6226):620-622.

98. Subramanian S, et al. Sustained expression of circulating human alpha- 1 antitrypsin reduces inflammation, increases $\mathrm{CD}^{+}{ }^{+} \mathrm{FoxP}^{+}{ }^{+}$Treg cell population and prevents signs of experimental autoimmune encephalomyelitis in mice. Metab Brain Dis. 2011;26(2):107-113.

99. Guo H, et al. An activated protein $\mathrm{C}$ analog stimulates neuronal production by human neural progenitor cells via a PAR1-PAR3-S1PR1-Akt pathway. J Neurosci. 2013;33(14):6181-6190. 